\title{
CHALLENGES IN PEDIATRIC INFLAMMATORY BOWEL DISEASE
}

\section{Mihai Larisia1,2, Dumitru Eugen1,2, Pruna Irina1,2, Chisnoiu Tatiana',2, Ungureanu Adina1, Chirila Sergiu', Mihai Gristina ${ }^{1,2}$, Balasa Adriana1,2}

${ }^{1}$ Faculty of Medicine, University "Ovidius" of Constanta

${ }^{2}$ Clinical Emergency County Hospital of Constanta

\section{ABSTRACT}

Inflammatory bowel disease (IBD) is a chronic condition of the gastrointestinal tract comprising of two entities: Crohn disease (CD) and Ulcerative colitis (UC). Considered rare in children in the past, inflammatory bowel disease is nowadays more frequently found, raising diagnostic and treatment challenges.

In our study, we have taken into consideration all children diagnosed with inflammatory bowel disease, in the Department of Pediatrics of the Clinical Emergency County Hospital of Constanta, from 2016 to 2019.

14 children were diagnosed with inflammatory bowel disease during this timeframe, 8 with Crohn disease $(57,14 \%)$ and 6 with Ulcerative colitis $(42,86 \%)$. The mean age at onset was 8.2 years for Crohn disease patients, varying between 20 months to 15 years, and 12.8 years for Ulcerative colitis patients, varying between 8 to 14 years.

After a positive diagnosis, different types of induction therapy was implemented, depending on the activity and severity of the disease, as well as on the type of inflammatory bowel disease. 12 patients received iv corticosteroids for an average of five days, followed by oral corticosteroids for 4-8 weeks. Aminosalicylates (Mesalazine) was used as a sole induction treatment in children with UC, or in association with corticosteroids in severe cases (4 cases). Immunomodulatory treatment (Azathioprine) was used for maintaining remission in 5 children with Crohn disease for an average period of 6 months. In 3 cases of CD, antibiotics, such as Metronidazole, were used in the initial treatment.

Biological therapy, such as Adalimumab (ADA), was administered as an induction therapy in patients with refractory to conventional treatment forms, in 5 cases, with a favourable outcome.

Keywords: children, Crohn disease, Ulcerative colitis.

\section{Introduction}

Inflammatory bowel disease (IBD) is a chronic condition of the gastrointestinal tract comprising of two entities: Crohn disease (CD) and Ulcerative colitis (UC). Considered rare in children in the past, inflammatory bowel disease is nowadays more frequently found, raising diagnostic and treatment challenges. It is considered now that one in four patients has had the onset of IBD in childhood (1). The onset of IBD during puberty has a great impact on children's lives, as it involves, in the majority of the cases, the psychological and endocrinological transition to adult life. Nevertheless, very early onset (VEO) of IBD cases, diagnosed in children less than 5 years of age, raises concerns about the new discovered treatment, which has yet to be proven by clinical trials, due to lack of scientific evidence of safety and efficiency in a group of children in which the known, ,classic” therapy medication hasn't really shown satisfactory results.

The aim of the study was to determine the types of inflamatory bowel disease diagnosed in the last 3 years in our pediatric department, their clinical manifestations at onset, the way to establish a positive diagnosis and disscuss the applied treatment. 


\section{Material and method}

We revised clinical record cards of children diagnosed with IBD in the Pediatric Department, of the Clinical Emergency County Hospital, from Constanta, Romania, in a three year-period, 20162019. We analysed demographic characteristics like age, sex, rural/urban living conditions, and also clinical manifestations, imagistic procedures used for positive diagnosis, histological findings and treatment methods.

\section{Results and discussions}

14 children were diagnosed with IBD during a 3-year period: 8 children with Crohn disease and 6 children with Ulcerative colitis. There were 8 boys ( 6 with $\mathrm{CD}$ and 2 with UC) and 6 girls ( 2 with $\mathrm{CD}$ and 4 with UC). All the children resided in urban area. The mean age at onset was 8.2 years for $\mathrm{CD}$ patients varying between 20 months to 15 years, and 12.8 years for UC patients, varying between 8 to 14 years.

Clinical manifestations at the onset of Crohn Disease were:

- chronic diarrhea (6 cases);

- difuse abdominal pain (5 cases);

- right lower quadrant pain, mimicking appendicitis (2 cases), transferred from the Pediatric Surgery Department, one of them after a surgical intervention for a suspicion of appendicitis, that postponed the colonoscopy procedure for one month;

- $\quad$ stunting linear growth (4 cases);

- weight loss - severe (25, respectively 30 $\mathrm{Kg}$ ) in 2 cases, moderate (between 3-6 kg) in 4 cases;

- $\quad$ anorexia (1 case);

- delayed puberty (1 case);

- perianal involvment manifested as rectal bleeding ( 3 cases), occult bleeding (1 case), fissures (1 case), abscesses and perianal fistulae ( 1 case), rectal tenesmus ( 4 cases);

- severe anemia (3 cases).

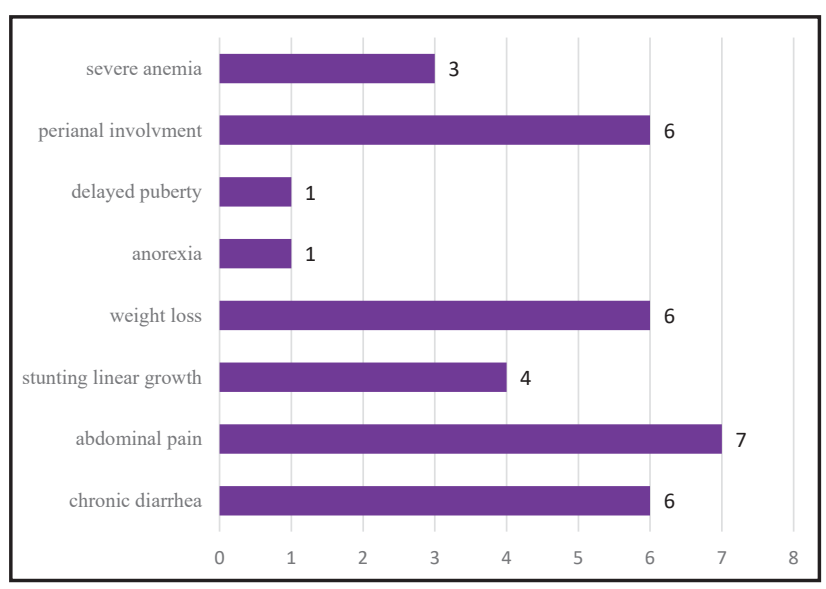

Figure 1 - Clinical manifestations frequency at the onset of Crohn disease

Clinical manifestations at onset of Ulcerative Colitis were:

- acute onset with diarrhea, rectal bleeding, tenesmus (3 cases) - previously admitted for investigations in an Infectious Disease Department;

- insidious onset with malnutrition and rectal bleeding (1 case);

- left upper abdominal quadrant pain, triggered by defecation (1 case);

- fever (2 cases);

- $\quad$ stunting linear growth (2 cases);

- weight loss (2 cases).

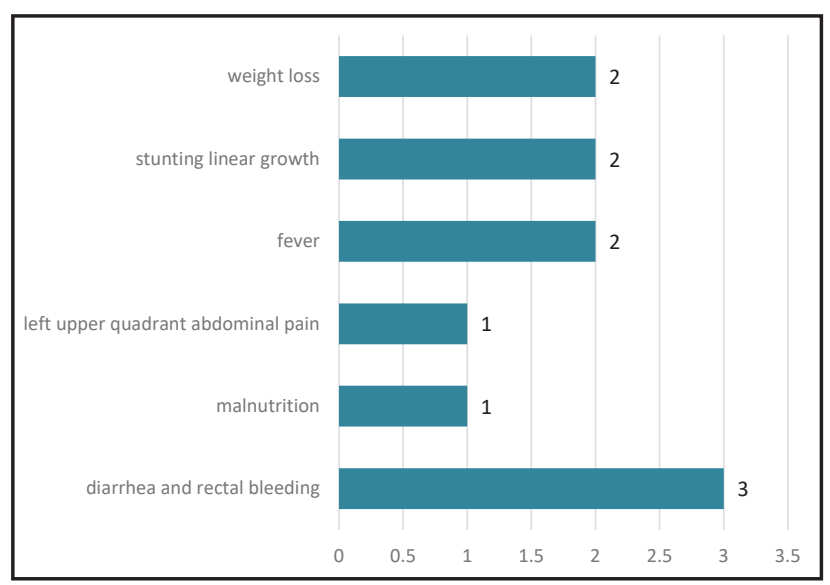

Figure 2-Clinical manifestations frequency at onset of Ulcerative colitis disease

In pediatric $\mathrm{CD}$, because of the right lower abdominal quadrant location of the pain, the diagnosis of appendicitis has to be ruled out. Ulcerative colitis symptoms overlap infectious colitis. Thus, UC should be suspicioned when symptoms persist, despite the antiinfectious treatment. 
Initial laboratory tests included complete blood cell count (CBC) with differential, inflammatory tests $(\mathrm{C}$ reactive protein level, ESR, fibrinogen), liver profile (serum alanine aminotransferase, serum aspartate aminotransferase, alkaline phosphatase, gamma glutamyl transpeptidase) albumin level, amylases, lipases.

Stool analysis included cultures, tests for occult haermorrhages. Clostridium difficile toxins A and B, Glutamat Dehydrogenase Test were negative in all the cases.

Fecal calprotectin was asked in every case at the onset and during evolution. We observed correlations between its values and clinical manifestations.

Quantiferon test was done in all the cases and repeated annualy.

Immunological analysis: pANCA and cANCA were asked in 6 cases, with negative results in most cases, except 1 case, where pANCA had positive values.

Imaging studies provided paramount information for positive diagnosis. All the children underwent ileocolonoscopy under general anesthesia at the onset of the disease, at 6 months and at need, depending on the clinical evolution.

$\mathrm{CD}$ endoscopic findings:

- distal ileum lesions, with proximal and distal extension;

- colonic localization;

- aphthoid ulcers;

- discontinuous inflammatory lesions, which frequently involve the ileocecal area;

- edematous mucosa, hyperemic, profound and superficial ulcers, which alternate with healthy mucosa areas.

Taking into account the endoscopic findings, from the $8 \mathrm{CD}$ cases, 3 were considered severe, with a Mayo endoscopic subscore of 3.

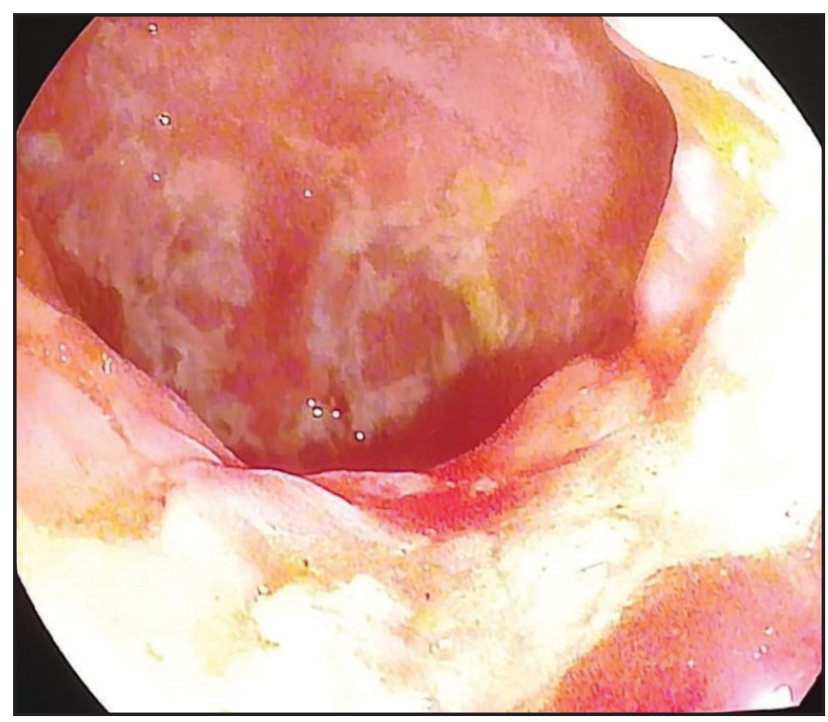

Figure 3 Erossive lesions, hyperemic and

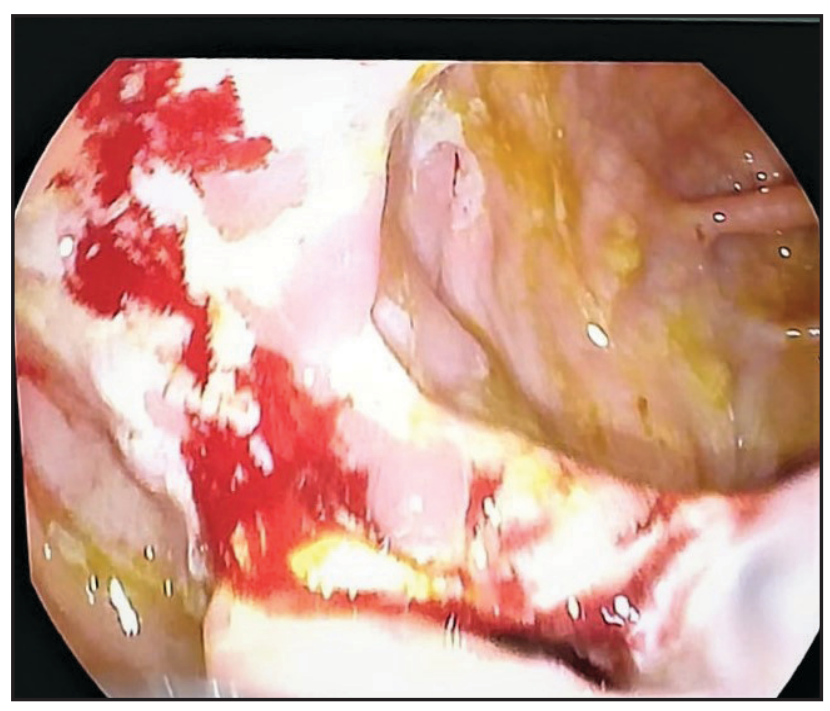

Figure 4 Extensive haemorrhagic lesions at edematous mucosa in the descending colon. cecum level.

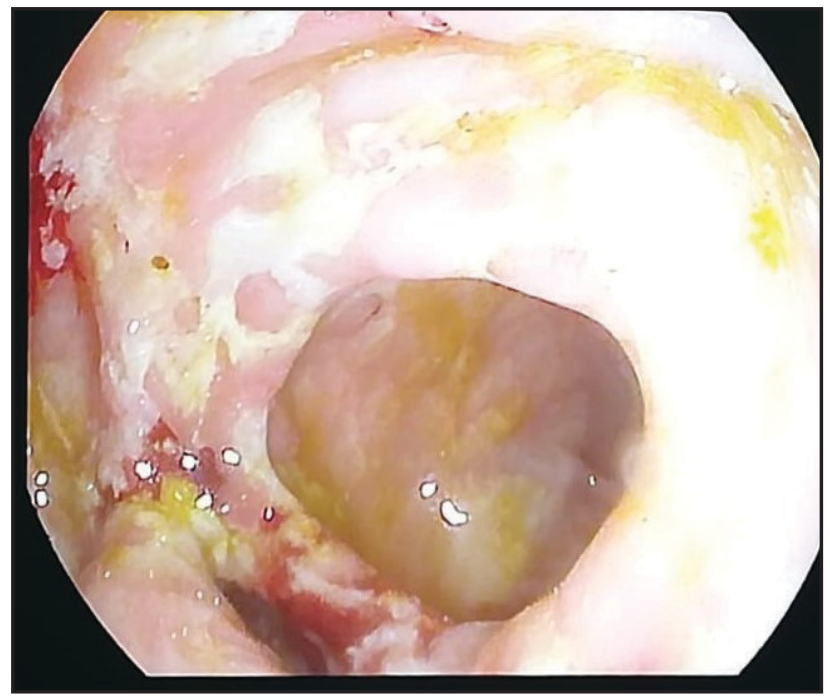

Figure 5 Ileal haemorrhagic lesions and edema. 




Figure 6 Rectal loss of vascular pattern and ulcerative lesions

UC endoscopic findings:

- rectal lesions, with varied degree progression in a difuse, continued pattern;

- difuse pancolitis with backwash ileitis (1 case);

- edematous mucosa, erythema, loss of vascular markings and mucosal friability;

- aphthoid type ulcerations.

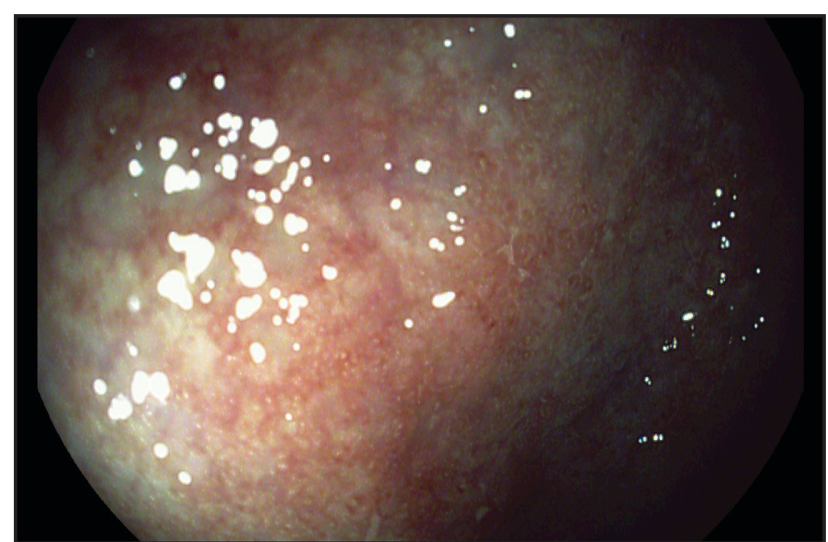

Figure 7 Rectal loss of vascular pattern aphthoid ulcerations

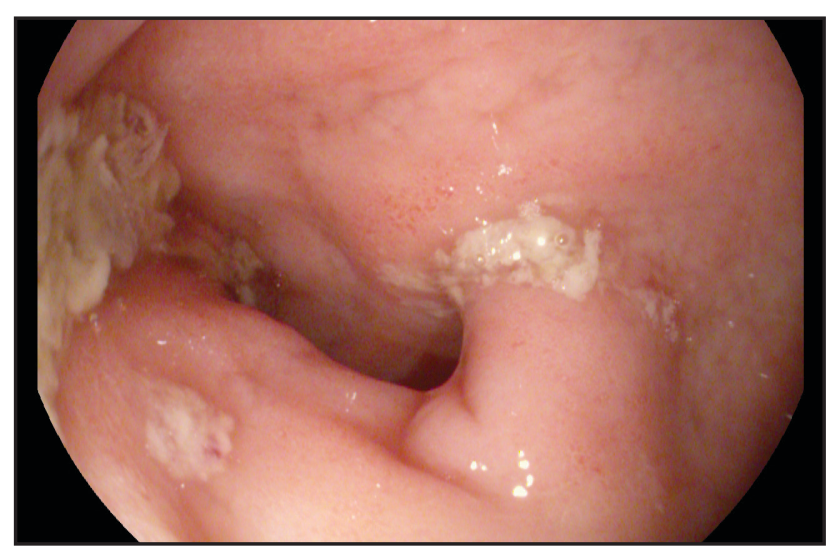

Figure 8 Rectal edema.
From the total of 6 cases if UC, 4 were given a Mayo endoscopic subscore of 3 (severe disease).

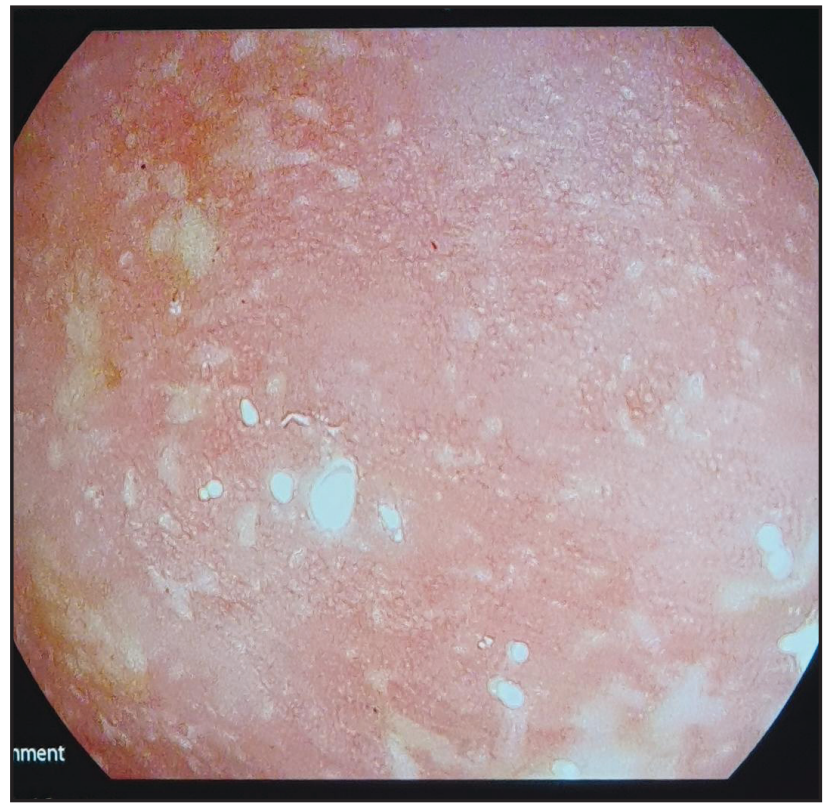

Figure 9. Continuous rectal mucosa edema and haemorrhage

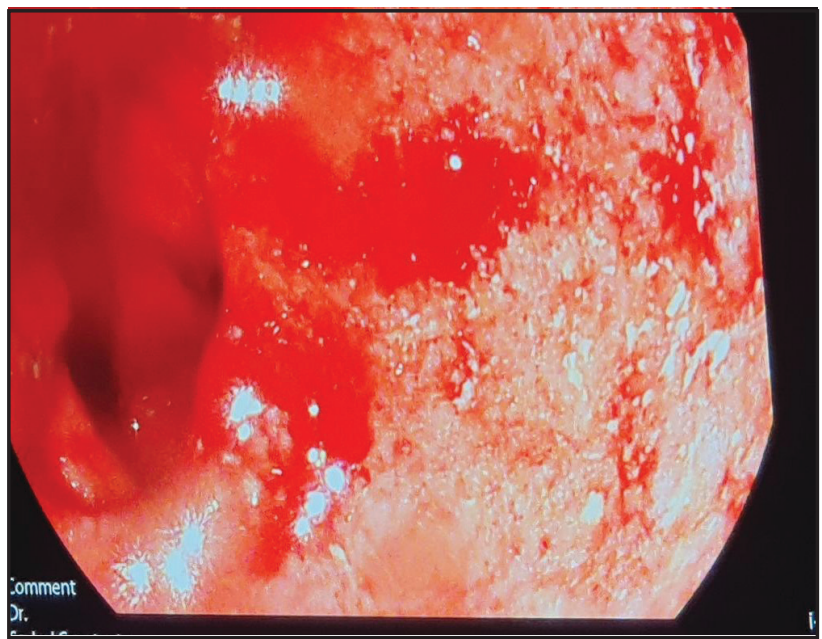

Figure 10. Rectal aphthoid lesions, loss of vascular pattern

Serial biopsies were obtained and histological results confirmed the diagnosis.

Crohn's disease histological findings were:

Transmural inflammation, fibrosis;

- Non caseous granuloma with giant multinuclated cells (62,5\% of CD cases);

- Mucosal ulcers with granulation tissue and fibrotic areas variable in depth;

- Cripts with architectural alterations - Goblet cells and rare Paneth cells;

- Chronic and acute inflammatory infiltrate. 
UC histological findings:

- Cript abscess (50\% of UC cases);

- Active criptitis;

- Chronic inflammation in the mucosa;

- Mucosal ulcers as a result of ongoing acute inflammatory process in severe cases;

- Cripts with architectural alterations - they can appear atrophic, shorter, wider, or have ramifications;

- Pseudopolypoid lesions;

- Chronic inflammatory infiltrate rich in plasmocytes, associated with eosinophilia and neutrophilia.

Non caseous granuloma with multinucleate giant cells is a specific lesion for $C D$, but inconsistenly present ( $60 \%$ of pediatric cases) (2). Crypt abscess is a characteristic lesion for UC but not specific (3).

To assess IBD severity and activity, corroborating clinical manifestations and endoscopic findings, the Mayo score as well as CDAI score were calculated, and taken into account for single or combined induction therapy.

Use of CT scan in order to determine if the patients have abscesses provided negative results.

Treatment: After the diagnosis, the goals of therapy consist of clinical remission, improving quality of life, restoring growth, and preventing complications, while minimizing the medication's side effects. (4)

Corticosteroids are indicated to induce remission in Crohn disease and Ulcerative Colitis. A number of 12 IBD patients received iv corticosteroids for an average of five days, followed by oral corticosteroids in tapered doses for 4-8 weeks.

Enteral nutrition therapy reffers to exclusive administration of liquid formula, for 8 weeks. It was completed by 5 children with Crohn disease in parallel with medication. According to Rossen et al, exclusive enteral nutrition (EEN) is as effective as corticosteroid therapy for inducing clinical remission in mild and moderate clinical forms (4). After it, we used the indication of the CD exclusive diet (CDED) phase II, thus providing $25 \%$ from the daily caloric requirement, a liquid formula, and specific foods from a list designed by a group of researchers of ESPGHAN. Now it is recommended that first phase of CDED should contain $50 \%$ of liquid formula and some mandatory foods. CDED targets the microbiome, not the inflammatory pathways, and is designed to reduce exposure to dietary components that have adverse effects on the microbiome and intestinal barrier (5). As Levine and colab showed in their article, CDED associated with partial enteral nutrition was better tolerated than EEN in children with mild to moderate disease, inducing remission after 6 weeks.

Aminosalicylates, such as Mesalazine 50 $\mathrm{mg} / \mathrm{kg} /$ day orally, enema or supository were used to treat Ulcerative Colitis. For the induction treatment corticosteroids were also associated in 4 children with severe forms.

Immunomodulatory treatment as Azathioprine 1,5-2 mg/kg/day was used for maintaining remission in 5 children with Crohn disease for an average period of 6 months. Children were followed for hepatotoxic and mielosuppressive side effects, that didn't appear in our study. However, in other studies, at the therapeutic dosage, hematologic toxicity appeared in $1,8 \%$ of the cases (6).

Antibiotics, such as Metronidazole, 10-20 $\mathrm{mg} / \mathrm{Kg} /$ day were used in the initial treatment of 3 children, including the child with Crohn disease related perianal fistulae.

Biological therapy. According to ECCO ESPGHAN consensus guidelines for Crohn disease, anti TNF medication is recommended for inducing and maintaining remission, despite prior optimized imunosupressive therapy, or for inducing remission in children with refractory corticosteroid treatment (7).

Adalimumab (ADA) was administered as an induction therapy in patients under $40 \mathrm{~kg}$ with $2,4 \mathrm{mg} / \mathrm{kg}$ as the first dose, followed by the same dose after two weeks, then $1,2 \mathrm{mg} / \mathrm{kg}$ every other week. We used Adalimumab in 5 patients, the most notable being the following:

TM, 7 years, severe ileocolonic Crohn disease, with lack of response to corticosteroids after 1 week, haemorrhagic shock, severe anemia, necessitating repeated red packed blood cells and plasma transfusions, started Adalimumab with $40 \mathrm{mg}-40 \mathrm{mg}-20 \mathrm{mg}$, continued with 20 mg every other week, with clinical and biological remission presently, after being 6 months into treament. 
MC, 15 years, severe ileocolonic Crohn disease, started the treatment with corticosteroids, for 12 weeks and azathioprine for 6 months, had several relapses, and was put on a $80 \mathrm{mg}-80$ $\mathrm{mg}-40 \mathrm{mg}$ treatment regimen of Adalimumab approximately 6 months ago, and has had favourable evolution.

LT, 8 years, diagnosed with IBD at 2 years, colonoscopy suggestive for Ulcerative pancolitis associating autoimmune colangitis, started treatment with corticosteroids and azathioprine, then Infliximab for 2 years, developing antiinfliximab antibodies, and loss of clinical, biological and mucosal response, had his treatment recently changed for a $80 \mathrm{mg}-40 \mathrm{mg}$ - $40 \mathrm{mg}$ regimen of Adalimumab.

\section{Eonclusions}

The rise in pediatric IBD observed in the last years, especially at very early onset ages, increases the challanges in diagnosis as well as treatment, due to the scarce data that allready exists in published clinical trials, especially in refractory to conventional treatment cases.

Although studies of anti-TNF medication administration in children have been made, there isn't enough information regarding long-term administration, efficiency and safety in children smaller than 6 years of age.

\section{References}

1. Aguirre A, Nugent CA. Oral Manifestation of Crohn's Disease. New England Journal of Medicine. 2015;373(13):1250.

2. Kugathasan S, Judd RH, Hoffmann RG, Heikenen J, Telega G, Khan F, et al. Epidemiologic and clinical characteristics of children with newly diagnosed inflammatory bowel disease in wisconsin: a statewide population-based study. The Journal of Pediatrics. 2003;143(4):525-31.

3. Bousvaros A, Antonioli D, Colletti R, Dubinsky M, Glickman J, Gold B, et al. Differentiating Ulcerative Colitis from Crohn Disease in Children and Young Adults: Report of a Working Group of the North American Society for Pediatric Gastroenterology, Hepatology, and Nutrition and the Crohn's and Colitis Foundation of America. Journal of pediatric gastroenterology and nutrition. 2007;44:653-74.

4. Rosen MJ, Dhawan A, Saeed SA. Inflammatory Bowel Disease in Children and Adolescents. JAMA Pediatrics. 2015;169(11):1053-60.

5. Levine A, Wine E, Assa A, Sigall Boneh R, Shaoul R, Kori M, et al. Crohn\&\#x2019; Disease Exclusion Diet Plus Partial Enteral Nutrition Induces Sustained Remission in a Randomized Controlled Trial. Gastroenterology. 2019;157(2):440-50.e8..

6. Hindorf $U$, Lindqvist $M$, Hildebrand $H$, Fagerberg U, Almer S. Adverse events leading to modification of therapy in a large cohort of patients with inflammatory bowel disease. Alimentary Pharmacology \& Therapeutics. 2006;24(2):331-42.

7. Ruemmele FM, Veres G, Kolho KL, Griffiths A, Levine A, Escher JC, et al. Consensus guidelines of ECCO/ESPGHAN on the medical management of pediatric Crohn's disease. Journal of Crohn's and Colitis. 2014;8(10):1179-207. 